\title{
Solving Fully Fuzzy Linear Systems with Trapezoidal Fuzzy Number Matrices by Partitioning
}

\author{
N.Jayanth Karthik \\ Department of Mathematics \\ D.G.Vaishnav College \\ Chennai 600106, India
}

\author{
E.Chandrasekaran \\ Department of Mathematics \\ Presidency College \\ Chennai 600005, India
}

\begin{abstract}
In this paper, a fully fuzzy linear system is solved by partitioning the coefficient matrix into sub matrices with trapezoidal fuzzy number matrices. The method is illustrated with numerical examples.
\end{abstract}

\section{General Terms}

Fuzzy Linear Systems.

\section{Keywords}

Fully Fuzzy Linear Systems, Trapezoidal fuzzy number matrices, partitioning, Schur complement.

\section{INTRODUCTION}

Linear system of equation has applications in many areas of science, engineering, finance and economics. Fuzzy linear system whose coefficient matrix is crisp and right hand side column is an arbitrary fuzzy number was first proposed by Friedman et al [4]. A linear system is called a fully fuzzy linear system (FFLS) if all coefficients in the system are all fuzzy numbers .Several direct and iterative methods based on numerical algorithms were used for solving fuzzy linear systems have been introduced by many authors $[1,2,3,8]$.

In this paper a FFLS is solved by partitioning the coefficient matrix into sub matrices with trapezoidal fuzzy numbers matrices. This paper is organized as follows. Some basic definitions and results on fuzzy sets and trapezoidal fuzzy numbers are given in section 2.In section 3, method to solve FFLS with trapezoidal fuzzy numbers matrices by partitioning is given. Illustrations with numerical examples are given in section 4 . Section 5 ends this paper with conclusion.

\section{PRELIMINARIES}

1. A fuzzy subset $\tilde{A}$ of $R$ is defined by its membership function by its membership function $\mu_{\tilde{\AA}}: \mathrm{R} \rightarrow[0,1]$ which assigns a real number $\mu_{\tilde{\AA}}$ in the interval $[0,1]$ to each element $\mathrm{X} \in \mathrm{R}$ where the value of $\mu_{\tilde{\mathrm{A}}}$ shows grade membership of $\mathrm{x}$ in $\tilde{A}$.

2. A trapezoidal fuzzy number denoted by $\tilde{\mathrm{A}}=(\mathrm{m}, \mathrm{n}, \alpha, \beta)$ has the membership function .

$$
\mu_{\tilde{\AA}}(\mathrm{x}) \quad\left\{\begin{array}{cc}
0 & \mathrm{x} \leq \alpha \\
\frac{\mathrm{x}-\alpha}{\mathrm{m}-\alpha} & \alpha \leq \mathrm{x} \leq \mathrm{m} \\
1 & \mathrm{~m} \leq \mathrm{x} \leq \mathrm{n} \\
\frac{\beta-\mathrm{x}}{\beta-\mathrm{n}} & \mathrm{n} \leq \mathrm{x} \leq \beta \\
0 & \mathrm{x} \geq \beta
\end{array}\right.
$$

3. A fuzzy number $\tilde{A}$ is called positive (negative) denoted by $\tilde{\mathrm{A}}>0(\tilde{\mathrm{A}}<0)$ if its membership function $\mu_{\tilde{\mathrm{A}}}(\mathrm{x})$ satisfies $\mu_{\tilde{\mathrm{A}}}=0$, $\forall \mathrm{x} \leq 0(\forall \mathrm{x} \geq 0)$ using its mean value, left and right spreads such a fuzzy number $\tilde{\mathrm{A}}$ is symbolically written as $\tilde{\mathrm{A}}=$ $(m, n, \alpha, \beta)$ is positive if and only if $m-\alpha \geq 0$ and $n-\beta \geq 0$

4. A Trapezoidal fuzzy number $\tilde{\mathrm{A}}=(\mathrm{m}, \mathrm{n}, \alpha, \beta)$ is said to be zero trapezoidal fuzzy number if and only if $m=0, n=0, \alpha=0$, $\beta=0$.

5.Two fuzzy numbers $\mathrm{M}=(\mathrm{m}, \mathrm{n}, \alpha, \beta)$ and $\mathrm{N}=(\mathrm{x}, \mathrm{y}, \gamma, \delta)$ are equal if and only if $\mathrm{m}=\mathrm{x}, \mathrm{n}=\mathrm{y}, \alpha=\gamma, \beta=\delta$.

6. For twofuzzy numbers $\mathrm{M}=(\mathrm{m}, \mathrm{n}, \alpha, \beta)$ and $\mathrm{N}=(\mathrm{x}, \mathrm{y}, \gamma, \delta)$ the operations extended addition, extended opposite and extended multiplication are $(\mathrm{m}, \mathrm{n}, \alpha, \beta) \oplus(\mathrm{x}, \mathrm{y}, \gamma, \delta)=(\mathrm{m}+\mathrm{x}, \mathrm{n}+\mathrm{y}, \alpha+\gamma, \beta+\delta)$

$$
\begin{aligned}
& -\mathrm{M}=-(\mathrm{m}, \mathrm{n}, \alpha, \beta)=(-\mathrm{m},-\mathrm{n}, \beta, \alpha) \\
& \text { If } \mathrm{M}>0, \mathrm{~N}>0 \text { then } \\
& (\mathrm{m}, \mathrm{n}, \alpha, \beta) \otimes(\mathrm{x}, \mathrm{y}, \gamma, \delta)=(\mathrm{mx}, \mathrm{ny}, \mathrm{m} \gamma+\mathrm{x} \alpha, \mathrm{n} \delta+\mathrm{y} \beta) \ldots . .(1)
\end{aligned}
$$

For scalar multiplication

$\lambda \otimes(\mathrm{m}, \mathrm{n}, \alpha, \beta)=\left\{\begin{array}{cc}\lambda \mathrm{m}, \lambda \mathrm{n}, \lambda \alpha, \lambda \beta & \lambda \geq 0 \\ \lambda \mathrm{m}, \lambda \mathrm{n},-\lambda \alpha,-\lambda \beta & \lambda<0\end{array}\right.$

7. A matrix $\tilde{\mathrm{A}}=\left(\tilde{\mathrm{a}}_{\mathrm{ij}}\right)$ is called a fuzzy matrix if each element of $\tilde{A}$ is a fuzzy number. A fuzzy matrix $\tilde{A}$ is positive denoted by $\tilde{\mathrm{A}}>0$ if each element of $\tilde{\mathrm{A}}$ is positive. Fuzzy matrix $\tilde{\mathrm{A}}=\left(\tilde{\mathrm{a}}_{\mathrm{ij}}\right)$ which is $\mathrm{n} \times \mathrm{n}$ matrix can be represented such that $\tilde{\mathrm{a}}_{\mathrm{ij}}=$ $\left(a_{i j}, b_{i j}, m_{i j}, n_{i j}\right)$ where $\tilde{A}=(A, B, M, N)$ where $A=\left(a_{i j}\right)$ $\mathrm{B}=\left(\mathrm{b}_{\mathrm{ij}}\right), \mathrm{M}=\left(\mathrm{m}_{\mathrm{ij}}\right), \mathrm{N}=\left(\mathrm{n}_{\mathrm{ij}}\right)$ are $\mathrm{n} \mathrm{x}$ crisp matrices. 
8. A square matrix $\tilde{\mathrm{A}}=\left(\tilde{\mathrm{a}}_{\mathrm{ij}}\right)$ is symmetric matrix if $\tilde{\mathrm{a}}_{\mathrm{ij}}=\tilde{\mathrm{a}}_{\mathrm{ji}} \forall$ $\mathrm{i}, \mathrm{j}$

9. Consider $\mathrm{n} x \mathrm{n}$ fuzzy linear system of equations

$$
\begin{aligned}
& \left(\tilde{\mathrm{a}}_{11} \otimes \tilde{\mathrm{x}}_{1}\right) \oplus\left(\tilde{\mathrm{a}}_{12} \otimes \tilde{\mathrm{x}}_{2}\right) \oplus \ldots \oplus\left(\tilde{\mathrm{a}}_{1 \mathrm{n}} \otimes \tilde{\mathrm{x}}_{\mathrm{n}}\right)=\tilde{\mathrm{b}}_{1 .} \\
& \left(\tilde{\mathrm{a}}_{21} \otimes \tilde{\mathrm{x}}_{1}\right) \oplus\left(\tilde{\mathrm{a}}_{22} \otimes \tilde{\mathrm{x}}_{2}\right) \oplus \ldots . . \oplus\left(\tilde{\mathrm{a}}_{2 \mathrm{n}} \otimes \tilde{\mathrm{x}}_{\mathrm{n}}\right)=\tilde{\mathrm{b}}_{2} .
\end{aligned}
$$

$$
\left(\tilde{\mathrm{a}}_{\mathrm{n} 1} \otimes \tilde{\mathrm{x}}_{1}\right) \oplus\left(\tilde{\mathrm{a}}_{\mathrm{n} 2} \otimes \tilde{\mathrm{x}}_{2}\right) \oplus \ldots \oplus\left(\tilde{\mathrm{a}}_{\mathrm{n}} \otimes \tilde{\mathrm{x}}_{\mathrm{n}}\right)=\tilde{\mathrm{b}}_{\mathrm{n} .}
$$

The matrix of the above equation is $\tilde{\mathrm{A}} \otimes \tilde{\mathrm{x}}=\tilde{\mathrm{b}}$ where coefficient matrix $\tilde{\mathrm{A}}=\left(\tilde{\mathrm{a}}_{\mathrm{ij}}\right)$ Where

$1 \leq \mathrm{i}, \mathrm{j} \leq \mathrm{n}$ is a $\mathrm{n} \mathrm{x}$ n fuzzy matrix and $\tilde{\mathrm{x}}_{\mathrm{j}}, \tilde{\mathrm{b}}_{\mathrm{j}} \in \mathrm{F}(\mathrm{R})$. This system is called Fully Fuzzy Linear System. (FFLS).

\section{SOLVING \\ FFLS \\ USING TRAPEZOIDAL FUZZY NUMBER MATRICES BY PARTITIONING.}

If in a given FFLS $\tilde{A} \otimes \tilde{x}=\tilde{b}$, the order of $\tilde{A}$ is very large then computing inverse becomes very difficult .The given FFLS is converted to equivalent crisp system, which is partitioned into sub matrices using Schur complement. It is easier to work with these sub matrices as their orders areconsiderably reduced.

For solving $\mathrm{n} x \mathrm{n}$ FFLS $\tilde{\mathrm{A}} \otimes \tilde{\mathrm{x}}=\tilde{\mathrm{b}}$ where $\tilde{\mathrm{A}}=(\mathrm{A}, \mathrm{B}, \mathrm{M}, \mathrm{N})$ , $\tilde{\mathrm{x}}=(\mathrm{x}, \mathrm{y}, \mathrm{z}, \mathrm{w})$ and $\tilde{\mathrm{b}}=(\mathrm{b}, \mathrm{g}, \mathrm{h}, \mathrm{k})$

$(\mathrm{A}, \mathrm{B}, \mathrm{M}, \mathrm{N}) \otimes(\mathrm{x}, \mathrm{y}, \mathrm{z}, \mathrm{w})=(\mathrm{b}, \mathrm{g}, \mathrm{h}, \mathrm{k}) . \mathrm{Using}(1)$

$(\mathrm{Ax}, \mathrm{By}, \mathrm{Az}+\mathrm{Mx}, \mathrm{Bw}+\mathrm{Ny})=(\mathrm{b}, \mathrm{g}, \mathrm{h}, \mathrm{k})$

$\mathrm{Ax}=\mathrm{b}$

$\mathrm{By}=\mathrm{g}$

$\mathrm{Az}+\mathrm{Mx}=\mathrm{h}$

$\mathrm{Bw}+\mathrm{Ny}=\mathrm{k}$

The crisp linear systems $\mathrm{Ax}=\mathrm{b}$ and $\mathrm{By}=\mathrm{g}$ can be partitioned into sub matrices using Schur complement such that

$$
\begin{aligned}
& {\left[\begin{array}{ll}
A_{11} & A_{12} \\
A_{21} & A_{22}
\end{array}\right]\left[\begin{array}{l}
x^{(1)} \\
x^{(2)}
\end{array}\right]=\left[\begin{array}{l}
b_{1} \\
b_{2}
\end{array}\right]} \\
& {\left[\begin{array}{ll}
B_{11} & B_{12} \\
B_{21} & B_{22}
\end{array}\right]\left[\begin{array}{l}
y^{(1)} \\
y^{(2)}
\end{array}\right]=\left[\begin{array}{l}
g_{1} \\
g_{2}
\end{array}\right]}
\end{aligned}
$$

here each $\mathrm{A}_{\mathrm{ij}}$ is a sub matrix of $\mathrm{A}$, if $\mathrm{A}_{11}$ is nonsingular and $B_{i j}$ is a sub matrix of $B$, if $B_{11}$ is non singular then considering (3.1) as simultaneous equations we obtain

$$
A_{11} x^{(1)}+A_{12} x^{(2)}=b_{1} \ldots .(4.1) A_{21} x^{(1)}+A_{22} x^{(2)}=b_{2}
$$

Eliminating $\mathrm{X}^{(1)}$ from (4.1) and substituting in (4.2) we get

$\mathrm{x}^{(1)}=\mathrm{A}_{11}^{-1}\left(\mathrm{~b}_{1}-\mathrm{A}_{12} \mathrm{x}^{(2)}\right)$

$$
\left(A_{22}-A_{21} A_{11}^{-1} A_{12}\right) x^{(2)}=\left[b_{2}-\left(A_{21} A_{11}^{-1}\right) b_{1}\right] \ldots .(5.2)
$$

Similarly

$\mathrm{y}^{(1)}=\mathrm{B}_{11}^{-1}\left(\mathrm{~g}_{1}-\mathrm{B}_{12} \mathrm{y}^{(2)}\right)$

$\left(\mathrm{B}_{22}-\mathrm{B}_{21} \mathrm{~B}_{11}{ }^{-1} \mathrm{~B}_{12}\right) \mathrm{y}^{(2)}=\left[\mathrm{g}_{2}-\left(\mathrm{B}_{21} \mathrm{~B}_{11}{ }^{-1}\right) \mathrm{g}_{1}\right] \ldots .(\mathbf{6 . 2})$ substituting $\mathrm{x}$ in (2.3) we get $\mathrm{z}$ and substituting $\mathrm{y}$ in (2.4) we get $w$.

\section{NUMERICAL EXAMPLES.}

In this section we apply the algorithm for solving FFLS of fuzzy trapezoidal numbers.

EXAMPLE (1) Consider the FFLS

$\left[\begin{array}{cc}(1,1,1,5) & (8,5,9,1) \\ (6,4,4,2) & (2,2,2,6)\end{array}\right]\left[\begin{array}{c}\left(\mathrm{x}_{1}, \mathrm{y}_{1}, \mathrm{z}_{1}, \mathrm{w}_{1}\right) \\ \left(\mathrm{x}_{2}, \mathrm{y}_{2}, \mathrm{z}_{2}, \mathrm{w}_{2}\right)\end{array}\right]=\left[\begin{array}{c}(23,33,62,45) \\ (46,24,70,66)\end{array}\right]$

Solution

$$
\begin{aligned}
& \mathrm{A}=\left[\begin{array}{ll}
1 & 8 \\
6 & 2
\end{array}\right], \mathrm{B}=\left[\begin{array}{ll}
1 & 5 \\
4 & 2
\end{array}\right], \mathrm{M}=\left[\begin{array}{ll}
1 & 9 \\
4 & 2
\end{array}\right], \mathrm{N}=\left[\begin{array}{ll}
5 & 1 \\
2 & 6
\end{array}\right] . \\
& \mathrm{b}=\left[\begin{array}{l}
23 \\
46
\end{array}\right], \mathrm{g}=\left[\begin{array}{l}
33 \\
24
\end{array}\right], \mathrm{h}=\left[\begin{array}{l}
62 \\
70
\end{array}\right], \mathrm{k}=\left[\begin{array}{l}
45 \\
66
\end{array}\right]
\end{aligned}
$$

Using (3.1) we get $\left[\begin{array}{ll}1 & 8 \\ 6 & 2\end{array}\right]\left[\begin{array}{l}x_{1} \\ x_{2}\end{array}\right]=\left[\begin{array}{l}23 \\ 46\end{array}\right]$

$A_{11}=1, A_{12}=8, A_{21}=6, A_{22}=2, b_{1}=23, b_{2}=46$

we get $x_{1}+8 x_{2}=23,6 x_{1}+2 x_{2}=46$

on solving $\mathrm{x}=\left(\begin{array}{l}7 \\ 2\end{array}\right)$

Using (3.2) we get $\left[\begin{array}{ll}1 & 5 \\ 4 & 2\end{array}\right]\left[\begin{array}{l}y_{1} \\ y_{2}\end{array}\right]=\left[\begin{array}{l}33 \\ 24\end{array}\right]$

$\mathrm{B}_{11}=1, \mathrm{~B}_{12}=5, \mathrm{~B}_{21}=4, \mathrm{~B}_{22}=2, \mathrm{~g}_{1}=33, \mathrm{~g}_{2}=24$

we get $y_{1}+5 y_{2}=33,4 y_{1}+2 y_{2}=24$ 
on solving $\mathrm{y}=\left(\begin{array}{l}3 \\ 6\end{array}\right)$

Using (2.3) we get $\left[\begin{array}{ll}1 & 8 \\ 6 & 2\end{array}\right]\left[\begin{array}{l}z_{1} \\ z_{2}\end{array}\right]+\left[\begin{array}{ll}1 & 9 \\ 4 & 2\end{array}\right]\left[\begin{array}{l}x_{1} \\ x_{2}\end{array}\right]=\left[\begin{array}{l}62 \\ 70\end{array}\right]$

substituting $\mathrm{x}_{1}, \mathrm{x}_{2}$ values we get $\mathrm{z}_{1}+8 \mathrm{z}_{2}=37$,

$6 z_{1}+2 z_{2}=38$, on solving $\mathrm{z}=\left(\begin{array}{l}5 \\ 4\end{array}\right)$

Using (2.4) we get $\left[\begin{array}{ll}1 & 5 \\ 4 & 2\end{array}\right]\left[\begin{array}{l}\mathrm{w}_{1} \\ \mathrm{w}_{2}\end{array}\right]+\left[\begin{array}{ll}5 & 1 \\ 2 & 6\end{array}\right]\left[\begin{array}{l}\mathrm{y}_{1} \\ \mathrm{y}_{2}\end{array}\right]=\left[\begin{array}{l}45 \\ 66\end{array}\right]$

substituting $\mathrm{y}_{1}, \mathrm{y}_{2}$ values we get $\mathrm{w}_{1}+5 \mathrm{w}_{2}=24$,

$4 \mathrm{w}_{1}+2 \mathrm{w}_{2}=24$ on solving $\mathrm{w}=\left(\begin{array}{l}4 \\ 4\end{array}\right)$

Hence the solution of the fully fuzzy linear system is

$\tilde{\mathrm{x}}=\left[\begin{array}{l}(7,3,5,4) \\ (2,6,4,4)\end{array}\right]$.

\section{EXAMPLE (2) Consider the FFLS}

$\left(\begin{array}{llll}(1,4,4,4) \quad(2,6,6,6) \quad(3,6,1,2) & (2,4,3,6)\end{array}\right)\left(\begin{array}{l}\left(\mathrm{x}_{1}, \mathrm{y}_{1}, \mathrm{z}_{1}, \mathrm{w}_{1}\right) \\ (2,6,6,6)\end{array}(8,18,4,3) \quad(10,36,172,160)\right.$

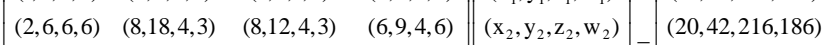

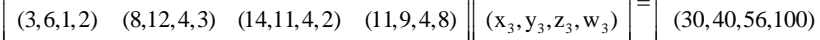

$\left(\begin{array}{llll}(2,4,3,6) & (6,9,4,6) & (11,9,4,8) & (10,13,2,3\end{array}\right)\left(\begin{array}{l}\left(\mathrm{x}_{3}, \mathrm{y}_{3}, \mathrm{z}_{3}, \mathrm{w}_{3}\right) \\ \left(\mathrm{x}_{4}, \mathrm{y}_{4}, \mathrm{z}_{4}, \mathrm{w}_{4}\right)\end{array}\right)\left(\begin{array}{c}(30,40,56,100) \\ (40,36,82,82)\end{array}\right)$

\section{Solution}

Since $\tilde{A}$ is symmetric A and B are symmetric and further $\mathrm{A}_{21}=\mathrm{A}_{12}{ }^{\mathrm{T}}$ and $\mathrm{B}_{21}=\mathrm{B}_{12}{ }^{\mathrm{T}}$.

$A=\left(\begin{array}{cccc}1 & 2 & 3 & 2 \\ 2 & 8 & 8 & 6 \\ 3 & 8 & 14 & 11 \\ 2 & 6 & 11 & 10\end{array}\right) \quad b=\left(\begin{array}{c}10 \\ 20 \\ 30 \\ 40\end{array}\right)$

$A_{11}=\left(\begin{array}{ll}1 & 2 \\ 2 & 8\end{array}\right), A_{12}=\left(\begin{array}{ll}3 & 2 \\ 8 & 6\end{array}\right), A_{21}=\left(\begin{array}{ll}3 & 8 \\ 2 & 6\end{array}\right)$,

$A_{22}=\left(\begin{array}{ll}14 & 11 \\ 11 & 10\end{array}\right), b_{1}=\left(\begin{array}{l}10 \\ 20\end{array}\right), b_{2}=\left(\begin{array}{l}30 \\ 40\end{array}\right)$,

Using (5.2) and

$\mathrm{x}^{(2)}=\left(\begin{array}{cc}5 / 4 & -1 \\ -1 & 1\end{array}\right)\left[\left(\begin{array}{l}30 \\ 40\end{array}\right)-\left(\begin{array}{ll}3 & 8 \\ 2 & 6\end{array}\right)\left(\begin{array}{cc}2 & -1 / 2 \\ -1 / 2 & 1 / 4\end{array}\right)\left(\begin{array}{c}10 \\ 20\end{array}\right)\right]=\left(\begin{array}{c}-20 \\ 20\end{array}\right)$

$x^{(1)}=\left(\begin{array}{cc}2 & -1 / 2 \\ -1 / 2 & 1 / 4\end{array}\right)\left(\left(\begin{array}{l}10 \\ 20\end{array}\right)-\left(\begin{array}{cc}3 & 2 \\ 8 & 6\end{array}\right)\left(\begin{array}{c}-20 \\ 20\end{array}\right)\right)=\left(\begin{array}{c}30 \\ 0\end{array}\right)$
$B=\left(\begin{array}{cccc}4 & 6 & 6 & 4 \\ 6 & 18 & 12 & 9 \\ 6 & 12 & 11 & 9 \\ 4 & 9 & 9 & 13\end{array}\right) \mathrm{g}=\left(\begin{array}{l}36 \\ 42 \\ 40 \\ 36\end{array}\right)$

$\mathrm{B}_{11}=\left(\begin{array}{cc}4 & 6 \\ 6 & 18\end{array}\right), \mathrm{B}_{12}=\left(\begin{array}{cc}6 & 4 \\ 12 & 9\end{array}\right), \mathrm{B}_{21}=\left(\begin{array}{cc}6 & 12 \\ 4 & 9\end{array}\right)$,

$\mathrm{B}_{22}=\left(\begin{array}{cc}11 & 9 \\ 9 & 13\end{array}\right), \mathrm{g}_{1}=\left(\begin{array}{l}36 \\ 42\end{array}\right), \mathrm{g}_{2}=\left(\begin{array}{l}40 \\ 36\end{array}\right)$

Using (6.2) and (6.1)

$$
\begin{aligned}
& \mathrm{y}^{(2)}=\left(\begin{array}{cc}
2 & -1 / 2 \\
-1 / 2 & 1 / 4
\end{array}\right)\left[\left(\begin{array}{l}
40 \\
36
\end{array}\right)-\left(\begin{array}{cc}
6 & 12 \\
4 & 9
\end{array}\right)\left(\begin{array}{cc}
1 / 2 & -1 / 6 \\
-1 / 6 & 1 / 9
\end{array}\right)\left(\begin{array}{c}
36 \\
42
\end{array}\right)\right]=\left(\begin{array}{c}
-22 \\
6
\end{array}\right) \\
& \mathrm{y}^{(1)}=\left(\begin{array}{cc}
1 / 2 & -1 / 6 \\
-1 / 6 & 1 / 9
\end{array}\right)\left(\left(\begin{array}{l}
36 \\
42
\end{array}\right)-\left(\begin{array}{cc}
6 & 4 \\
12 & 9
\end{array}\right)\left(\begin{array}{c}
-22 \\
6
\end{array}\right)\right)=\left(\begin{array}{c}
30 \\
4
\end{array}\right)
\end{aligned}
$$

substituting $\mathrm{x}$ in (2.3) we get $\mathrm{z}$

$\mathrm{z}=\left(\begin{array}{cccc}4 & -1 / 4 & -3 / 2 & 1 \\ -1 / 4 & 5 / 16 & -1 / 8 & 0 \\ -3 / 2 & -1 / 8 & 5 / 4 & -1 \\ 1 & 0 & -1 & 1\end{array}\right)\left(\left(\begin{array}{c}172 \\ 216 \\ 56 \\ 82\end{array}\right)-\left(\begin{array}{cccc}4 & 6 & 1 & 3 \\ 6 & 4 & 4 & 4 \\ 1 & 4 & 4 & 4 \\ 3 & 4 & 4 & 2\end{array}\right)\left(\begin{array}{c}30 \\ 0 \\ -20 \\ 20\end{array}\right)\right)=\left(\begin{array}{c}32 \\ 5 \\ -22 \\ 18\end{array}\right)$

substituting y in (2.4) we get w.

$\mathrm{w}=\left(\begin{array}{cccc}33 / 16 & 7 / 24 & -7 / 4 & 3 / 8 \\ 7 / 24 & 1 / 4 & -1 / 2 & 1 / 12 \\ -7 / 4 & -1 / 2 & 2 & -1 / 2 \\ 3 / 8 & 1 / 12 & -1 / 2 & 1 / 4\end{array}\right)\left(\left(\begin{array}{c}160 \\ 186 \\ 100 \\ 82\end{array}\right)-\left(\begin{array}{llll}4 & 6 & 2 & 6 \\ 6 & 3 & 3 & 6 \\ 2 & 3 & 2 & 8 \\ 6 & 6 & 8 & 3\end{array}\right)\left(\begin{array}{c}30 \\ 4 \\ -22 \\ 6\end{array}\right)\right)=\left(\begin{array}{c}28 \\ 4 \\ -24 \\ 8\end{array}\right)$

Hence the solution of the fully fuzzy linear system is

$\tilde{\mathrm{x}}=\left(\begin{array}{c}(30,30,32,28) \\ (0,4,5,4) \\ (-20,-22,-22,-24) \\ (20,6,18,8)\end{array}\right)$.

\section{CONCLUSION}

In this paper solution of FFLS $\tilde{A} \otimes \tilde{\mathrm{x}}=\tilde{\mathrm{b}}$ is obtained by partitioning the coefficient matrix into sub matrices using Schur complement by a new methodology in the form of trapezoidal fuzzy number matrices. This method is very efficient for the system $\tilde{A} \otimes \tilde{x}=\tilde{b}$ whenthe order of $\tilde{A}$ is very large.This method is easy to implement in parallel computing. 


\section{REFERENCES}

[1] Allahviranloo.T,Mikaeilvand.N 2009, Solutions of fully fuzzy linear system ,Proceedings International conference on boundary value problem : mathematical model in Engineering, Biology and Medicine, Volume $1124,234-243$.

[2] Amitkumar, AbinavBansal, Neetu 2010, A method for solving fully fuzzy linear system with trapezoidal fuzzy numbers , Iranian journal of optimization 2, 359-374.

[3] Dehghan.M , Hashemi.B, Ghatee.M, 2007, Solution of fully fuzzy linear systems using iterative techniques, Applied Mathematics and Computation, Volume 32 , Issue 2 , 316-336

[4] Friedman.M , Ming.M ,Kandel.A , 1998, Fuzzy linear systems , Fuzzy sets and systems Volume 96 , 201-209

[5] Hashemi.M.S ,Mirnia.M.K and Shahmorad.S 2008. Solving fuzzy linear systems by using schur complement when coefficient matrix is an m-matrix , Iranian journal of fuzzy systems, Volume 5 , No 3, 15-29

[6] Mansouri.P and Asady.T, 2011, Iterative methods for solving fuzzy linear systems , Australian Journal of basic and applied sciences 5(7) 1036-1049.

[7] Meenakshi A.R. and Jenita.P , 2010 , On k-Regularity of block fuzzy matrices, International journal of Math. Sciences volume 52010 no $24,1169-1176$.

[8] Nasseri. S.H.,Gholami .M , 2011 , Linear system of equations with trapezoidal fuzzy numbers, The Journal of Mathematics and Computer science Volume 3,71-79.x

[9] Zhang.F,2005 The Schur complement and its applications ,Springer 